\title{
Electron interaction, charging, and screening at grain boundaries in graphene
}

\author{
S Ihnatsenka and Igor Zozoulenko
}

\section{Linköping University Post Print}

\section{Tweet}

N.B.: When citing this work, cite the original article.

Original Publication:

S Ihnatsenka and Igor Zozoulenko, Electron interaction, charging, and screening at grain boundaries in graphene, 2013, Physical Review B. Condensed Matter and Materials Physics, (88), 8.

http://dx.doi.org/10.1103/PhysRevB.88.085436

Copyright: American Physical Society http://www.aps.org/ 


\title{
Electron interaction, charging, and screening at grain boundaries in graphene
}

\author{
S. Ihnatsenka* \\ Department of Physics, Simon Fraser University, Burnaby, British Columbia, Canada V5A IS6 \\ I. V. Zozoulenko ${ }^{\dagger}$ \\ Organic Electronics, Department of Science and Technology (ITN), Linköping University, 60174 Norrköping, Sweden
}

(Received 28 May 2013; published 28 August 2013)

\begin{abstract}
Electronic, transport, and spin properties of grain boundaries (GBs) are investigated in electrostatically doped graphene at finite electron densities within the Hartree and Hubbard approximations. We demonstrate that depending on the character of the GBs, the states residing on them can have a metallic character with a zero group velocity or can be fully populated losing the ability to carry a current. These states show qualitatively different features in charge accumulation and spin polarization. We also demonstrate that the semiclassical Thomas-Fermi approach provides a satisfactory approximation to the calculated self-consistent potential. The conductance of GBs is reduced due to enhanced backscattering from this potential.
\end{abstract}

DOI: 10.1103/PhysRevB.88.085436

PACS number(s): 81.05.ue, 72.10.Fk, 72.80.Vp, 73.22.Pr

\section{INTRODUCTION}

During recent years chemical vapor deposition (CVD) on transition metals has emerged as one of the most attractive methods for scalable graphene production. ${ }^{1,2}$ The advantages of this method is in its low cost, the possibility to grow large graphene sheets (tens of inches), and the ease of its transfer onto other substrates. Due to features of the growth process, CVD-grown graphene is polycrystalline, consisting of grains of various crystal orientations separated by one-dimensional extended line defects representing grain boundaries (GBs) ${ }^{3-5}$ Significant evidence has accumulated by now that the GBs strongly affect electrical transport ${ }^{6-11}$ and represent the limiting scattering mechanism of the electronic mobility in CVDgrown graphene. ${ }^{12-15}$ This provides a strong motivation for investigation of morphological, electronic, and spin properties of GBs. A number of studies have been recently reported addressing the band structure, ${ }^{16-20}$ spin polarization, ${ }^{16,17,21,22}$ electron transport and scattering ${ }^{12,13,20}$ in GBs. However, all these studies were limited to the case of electrically neutral graphene, and very little is presently known on how the electronic and transport properties of GBs are modified at nonzero electron densities (i.e., away from the Dirac point). At the same time, the effect of a finite electron density is of the utmost importance for the understanding of electron scattering by the GBs. Indeed, due to filling of quasibound states residing on them by electrons from the bulk, the GBs transform into charged lines which are believed to be responsible for the impediment of electron transport in CDV-grown graphene. Note that a local self-doping of individual GBs (i.e., transfer of electrons from the bulk to the states at GBs in a nominally neutral sample) has been recently observed by means of scanning tunneling microscopy (STM) measurements; ${ }^{9}$ it has also been argued that by doping by electrons from the bulk, the GBs can act as quasidimensional metallic wires. ${ }^{13,20,23}$ It is noteworthy that quasi-one-dimensional localized states of a related nature can reside on domain walls, ${ }^{24}$ graphene nanoroads, ${ }^{25}$ and $p$ - $n$ junctions in bilayer graphene. ${ }^{26}$

In the present work we depart from a conventional model of neutral graphene at the half filling and investigate how electronic and transport properties of GBs are affected by the presence of interacting electrons. We discuss how the charging of GBs evolves with the electron density and compare our findings with a semiclassical Thomas-Fermi (TF) model of screening. We also demonstrate that charging at finite electron densities leads to qualitatively new features in the band structure, and transport properties of grain boundary are strongly modified in a comparison to the noninteracting description.

\section{BASICS}

In graphene, GBs represent one-dimensional dislocations defined by interfaces between two domains of material with different crystallographic orientations. The latter are characterized by the angles $\theta_{L}$ and $\theta_{R}$ between the corresponding crystallographic directions in two domains and normal to the boundary line (Fig. 1). (See also an illustration in Fig. 6 in the Appendix.) The periodicity of the dislocation is defined by the translation vectors $(n, m)$ of the length $d$ belonging to the crystalline domains and oriented along the boundary line. In the present study we consider two representative GBs, $(2,0)$ and $(2,1)$, shown, respectively, in Figs. 1(a) and 1(b). The first one, $(2,0)$, consists of domains with the aligned crystallographic orientations $\theta_{L}=\theta_{R}=\theta=0^{\circ}(d \approx 0.5 \mathrm{~nm})$ and separated by a zigzag-oriented interface of one octagon and two side-sharing pentagons. ${ }^{16-19,23}$ The repeat vector $(2,1)$ of the second one implies $\theta_{L}=\theta_{R}=\theta=10.9^{\circ}(d \approx$ $0.65 \mathrm{~nm}$ ) and its interface region includes pentagon-heptagon pairs. ${ }^{6,17}$ We would like to note that while we study two representative GBs, $(2,0)$ and $(2,1)$ (corresponding to aligned and misaligned crystallographic orientations), we believe that our findings are generic and remain valid for other GBs in graphene.

For the case of spinless electrons we use a standard $p$-orbital tight-binding Hamiltonian, ${ }^{27}$

$$
H_{0}=\sum_{\mathbf{r}} V_{\mathbf{r}}^{\text {Hartree }} a_{\mathbf{r}}^{+} a_{\mathbf{r}}-\sum_{\mathbf{r}, \mathbf{r}+\Delta} t_{\mathbf{r}, \mathbf{r}+\Delta} a_{\mathbf{r}}^{+} a_{\mathbf{r}+\Delta},
$$

where $V_{\mathbf{r}}^{\text {Hartree }}$ is the Hartree potential at the site $\mathbf{r}$ and $t_{\mathbf{r}, \mathbf{r}+\Delta}$ is the overlap integral between the neighboring sites $\mathbf{r}$ and $\mathbf{r}+\Delta$. 
(a)
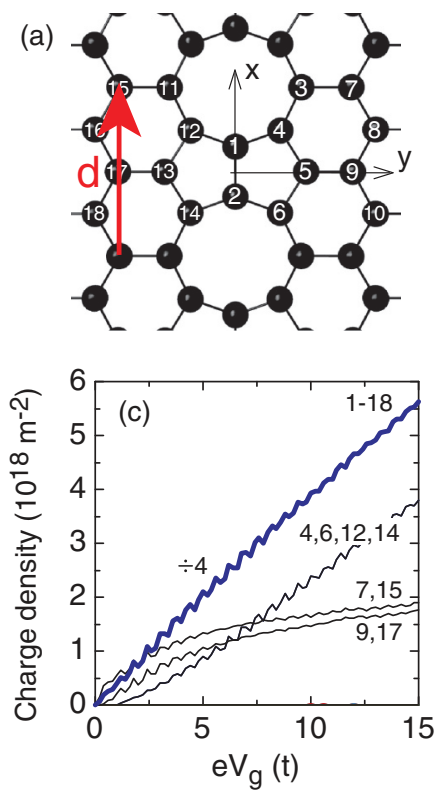

(b)
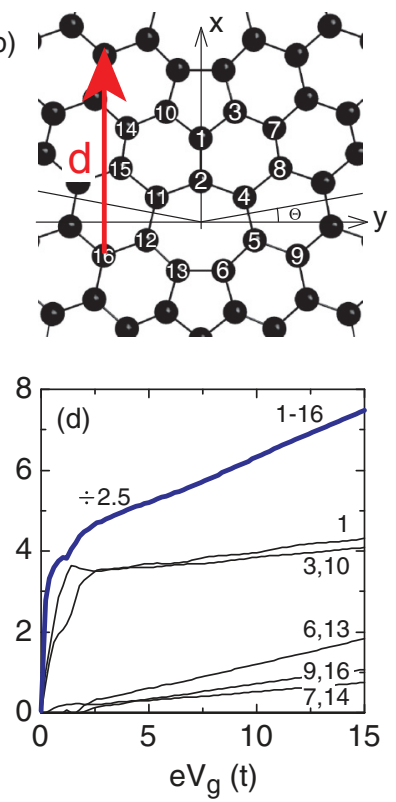

FIG. 1. (Color online) Atomic geometries of $(2,0) \mathrm{GB}$ and $(2,1)$ GB [panels (a) and (b), respectively]; the arrows correspond to the repeated vectors $(2,0)$ and $(2,1)$. Thick blue lines in panels (c) and (d) show the total occupancies for $(2,0)$ and $(2,1)$ GBs as a function of $E_{F}=e V_{g}$ (divided respectively by factors of 4 and 2.5). Thin black lines show occupancies of individual carbon atoms [enumerated in panels (a) and (b)].

The Hartree potential results from the Coulomb interaction between extra charges in the system,

$$
V_{\mathbf{r}}^{\text {Hartree }}=\frac{e^{2}}{4 \pi \varepsilon_{0} \varepsilon_{r}} \sum_{\mathbf{r}^{\prime} \neq \mathbf{r}} n_{\mathbf{r}^{\prime}}\left(\frac{1}{\left|\mathbf{r}-\mathbf{r}^{\prime}\right|}-\frac{1}{\sqrt{\left|\mathbf{r}-\mathbf{r}^{\prime}\right|^{2}+4 b^{2}}}\right),
$$

where $n_{\mathbf{r}^{\prime}}$ is the local electron occupation and the second term in the parentheses corresponds to the mirror charges. ${ }^{27}$ (In our calculation we assume that a graphene sheet is separated from a back gate by a dielectric of width $b=50 \mathrm{~nm}$ with the relative permittivity $\varepsilon_{r}=3.9$ ). The summation in the Hartree potential, Eq. (2), runs over the entire ribbon. In order to calculate $t_{\mathbf{r}, \mathbf{r}+\Delta}$ for the GBs studied in this paper we performed $a b$ initio geometry relaxations based on the density functional theory using the GAUSSIAN 09 software package. ${ }^{28}$ Away from the $\mathrm{GB}, t_{\mathbf{r}, \mathbf{r}+\Delta}=t=2.7 \mathrm{eV}$. Details of computations and the calculated values of the transfer integrals are presented in the Appendix. The number of excess electrons at site $\mathbf{r}$ reads $n_{\mathbf{r}}=\int_{-\infty}^{\infty} \rho(\mathbf{r}, E) f_{F D}\left(E, E_{F}\right) d E-n_{\text {ions }}$, where $\rho(\mathbf{r}, E)$ is the energy-dependent local density of states, $f_{F D}\left(E, E_{F}\right)$ is the Fermi-Dirac distribution function, $E_{F}=e V_{g}$ is the Fermi energy the value of which is adjusted by the gate voltage $V_{g}$, and $n_{\text {ions }}=3.8 \times 10^{19} \mathrm{~m}^{-2}$ is the positive charge background of ions. The Bloch states, the electron densities, and the band structure are calculated self-consistently using the Green's function technique as described in Refs. 27 and 29. The conductance calculations with a self-consistent potential are performed on the basis of the Landauer formalism using the standard recursive Green's function technique as described in Ref. 30. The band structure calculations are performed in the ribbon geometry with the GB residing in the middle of the

ribbon, which is infinite in the $x$ direction and has a finite width of $20 \mathrm{~nm}$ in the transverse $y$ direction.

For the case of electrons with spin $(\sigma=\uparrow, \downarrow)$ we introduce spin-dependent electron densities $n_{\mathbf{r}}^{\sigma}$ and use the same formalism as described above with a Hubbard Hamiltonian of the form $H=H^{\uparrow}+H^{\downarrow}, 29,31$

$$
H^{\sigma}=H_{0}+V_{\mathrm{Hubb}}^{\sigma} ; \quad V_{\mathrm{Hubb}}^{\sigma}=U n_{\mathbf{r}}^{\sigma^{\prime}},
$$

where $H_{0}$ is given by Eq. (1) and the Hubbard constant $U=t .^{32}$

\section{RESULTS AND DISCUSSION}

We start with the case of spinless electrons described by the Hamiltonian (1). Figures 2(a)-2(c) show the band structures of the $(2,0) \mathrm{GB}$ for different gate voltages $V_{g}$. For $V_{g}=0$ [Fig. 2(a)] the system remains neutral and the results of the Hartree approach correspond to the case of noninteracting electrons. The flat band at $E-E_{F}=0$ is nearly degenerate and corresponds to three states, one residing at the GB and two at the zigzag edges of the ribbon. (Note that even though the zigzag edge states contribute to the band diagram, we verified, by choosing a larger computational domain, that their overall effect on electrostatics and electronic properties of the state at the GB is negligibly small.) An inspection of the wave functions shows that the flat band of the GB is associated with an exponentially localized Bloch state which originates from the zigzag topology of the interface similar to the zigzag edges of zigzag ribbons. As $V_{g}$ increases the band structure changes substantially [see Figs. 2(b) and 2(c)]. The most distinct feature of the band diagram is that the state at the GB gets pinned to $E_{F}$ and remains partially filled at any $V_{g}$. This is because this state

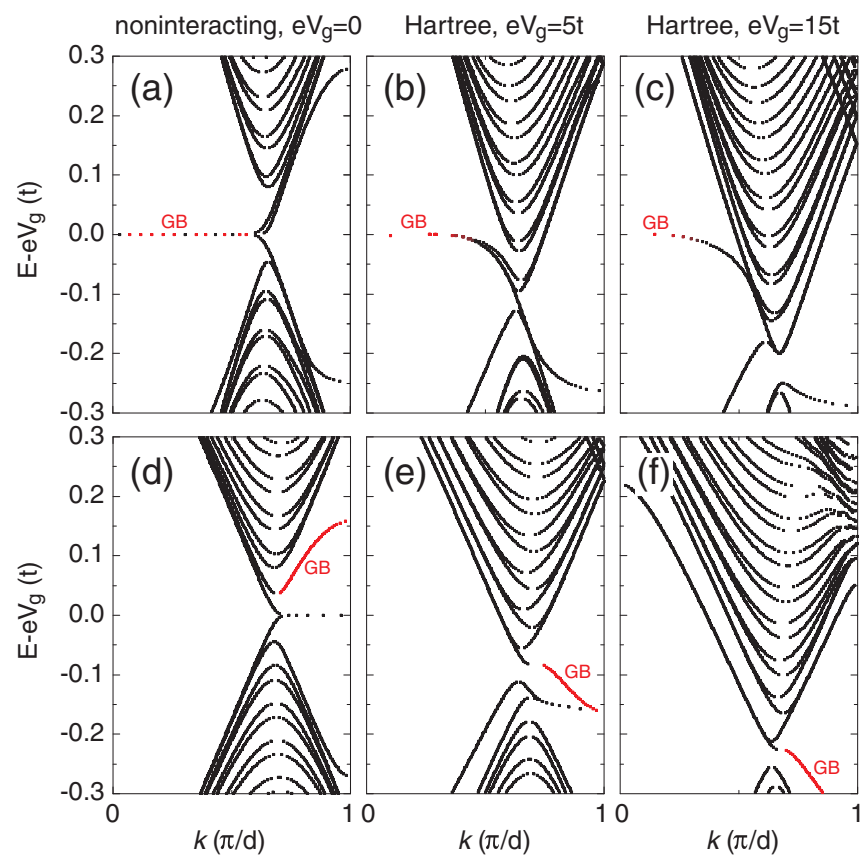

FIG. 2. (Color online) Evolution of the band structure of the $(2,0)$ GB state [panels (a)-(c)] and the (2,1) GB state [panels (d)-(f)] upon change of the gate voltage $V_{g}$ calculated in the Hartree approximation using Hamiltonian Eq. (1). States residing at GBs are drawn in red and marked by "GB." 
is flat and therefore has a high density of states (DOS). As a result, electrons filling this state can easily screen the external potential, which results in metallic behavior and pinning.

The state residing at the $(2,1)$ GB also shows exponential localization. However, features and evolution of the corresponding band in the dispersion relation are different from those of the $(2,0)$ state [see the state marked "GB" in Figs. 2(d)-2(f)]. At $V_{g}=0$ the state residing at the GB is practically empty as it lies above $E_{F}$ [Fig. 2(d)]. In contrast to the $(2,0)$ state, this state does not have a metallic character with a high DOS, and therefore it cannot screen the applied potential. Hence, with application of $V_{g}$ the $(2,1)$ GB state gets quickly populated, and a corresponding dispersion curve bends down and moves below $E_{F}$ [see Figs. 2(e) and 2(f)]. Note that a flat band at $E_{F}=0$ in Fig. 2(d) corresponds to the edge states of the zigzag nanoribbon.

Let us now discuss charge accumulation at the GBs due to the filling of the quasibound states residing on them. Figure 1 shows that the charge density at the $(2,0)$ GB grows linearly with an increase of the gate voltage $V_{g}$. In contrast, the charge density at the $(2,1)$ GB stays practically constant showing only a slow increase following the increase of the overall electron density in the ribbon as $V_{g}$ grows. This difference in the charge accumulation can be traced to different behavior of the band dispersions of the states residing at the $(2,0)$ and $(2,1)$ GBs discussed above. The linear charge accumulation on the $(2,0) \mathrm{GB}$ occurs because the corresponding state has a metallic character with a high DOS and therefore it remains only partially filled. As a result, an increase of the gate voltage leads to a gradual population of this state. On the contrary, with application of the gate voltage the state residing at the $(2,1) \mathrm{GB}$ becomes practically fully populated and immediately moves below $E_{F}$. Hence, a further increase of $V_{g}$ has very little effect on the charge accumulated at the $(2,1) \mathrm{GB}$. It should be noted that for both types of GBs considered here the local density of states (LDOS) and therefore the accumulated charge strongly depend on a site position [see Figs. 1(c) and 1(d)].

As mentioned in the Introduction, scattering at charged line defects is regarded as the limiting factor for the mobility in CVD graphene. An expression for the scattering potential can be obtained within the semiclassical TF approximation describing the screening of an extended charged line defect by the surrounding electron gas, ${ }^{12,15}$

$$
\begin{aligned}
V_{\mathrm{TF}}(x)= & \frac{\lambda e}{2 \pi \varepsilon_{0} \varepsilon_{\mathrm{r}}}\left\{-\cos \left(q_{\mathrm{TF}} x\right) \operatorname{Ci}\left(q_{\mathrm{TF}} x\right)\right. \\
& \left.+\sin \left(q_{\mathrm{TF}} x\right)\left[\frac{\pi}{2}-\operatorname{Si}\left(q_{\mathrm{TF}} x\right)\right]\right\},
\end{aligned}
$$

where $\mathrm{Ci}$ and $\mathrm{Si}$ denote the cosine and sine integral functions, $\lambda$ is the line charge density, and $q_{\mathrm{TF}}=e^{2} k_{F} /\left(\pi \varepsilon_{0} \varepsilon_{\mathrm{r}} \hbar v_{F}\right)$ is the TF wave vector defined by the electron Fermi velocity $v_{F}=3 \mathrm{ta} /(2 \hbar)$ and the Fermi momentum $k_{F}=\sqrt{\pi n}(a=$ $0.142 \mathrm{~nm}$ is the C-C distance). The TF potential (4) was used for semiclassical Boltzmann and quantum-mechanical Kubo calculations of the conductivity of CVD graphene. ${ }^{12,15}$ It is therefore important to find whether this potential provides a reliable approximation for the numerically exact quantummechanical self-consistent potential. A comparison between the TF potential (4) and the self-consistent potential calculated

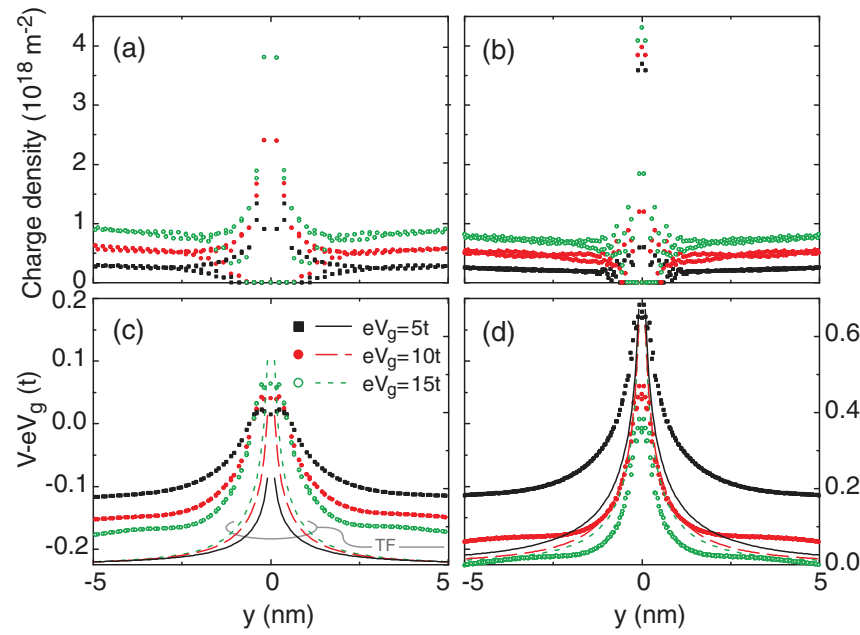

FIG. 3. (Color online) The charge density [panels (a) and (b)] and the potential [panels (c) and (d)] in a cross section of the ribbon calculated in the Hartree approximation for, respectively, $(2,0)$ and $(2,1) \mathrm{GBs}$ for different applied gate voltages $e V_{g}=5 t, 10 t$, and $15 t$. Thin solid, dashed, and dotted lines in panels (c) and (d) illustrate the results of the Thomas-Fermi approximation [Eq. (4)]. Extracted linear charge densities at GBs and the bulk electron densities (i.e., away from the GBs) are $\lambda / a=1.3,2.6,3.9 \times 10^{18} \mathrm{~m}^{-2}$ and $n=3,6,9 \times$ $10^{17} \mathrm{~m}^{-2}$ for panel (c) and $\lambda / a=3.7,4,4.3 \times 10^{18} \mathrm{~m}^{-2}$ and $n=2.5$, $5,7.5 \times 10^{17} \mathrm{~m}^{-2}$ for panel (d). Energy scale for the TF potentials (in units of $t$ ) is on the right of panels (c) and (d).

on the basis of the lattice Hamiltonian Eq. (1) is shown in Figs. 3(c) and 3(d) for, respectively, $(2,0)$ and $(2,1)$ GBs. In this comparison the electron density $n$ and the line charge density $\lambda$ in the TF potential [entering Eq. (4) as phenomenological parameters] have been extracted from our numerical calculations (see the caption to Fig. 3 ). The overall agreement for the potential height and width between the TF and the numerically exact potential is satisfactory. However, the TF approach predicts a potential that is more narrow and decays more rapidly in comparison to the exact one, especially for the $(2,0) \mathrm{GB}$. This can be related to a finite extent of the wave function disregarded in the TF approach where a state residing at a GB is treated as a charged line of a zero width. Note that because of the finite width of the ribbon the long-distance behavior of the exact self-consistent potential is obscured by edge effects. This makes it difficult to provide a quantitative comparison of its long-distance asymptotic to that of the TF potential (4) which decays as $V_{\mathrm{TF}}(x) \propto\left(q_{\mathrm{TF}} x\right)^{-2}$.

It has been speculated in the literature that GBs can be used as a one-dimensional quantum wire or a device component to carry the current in bulk graphene. ${ }^{13,20,23}$ Our findings suggest that these predictions might be too optimistic. Indeed, GB states with a flat dispersion [such as $(2,0)$ states] remain metallic and pinned at $E_{F}$ even at finite gate voltages when graphene is electrostatically doped by electrons. However, the group velocity of such states is practically zero, which makes these states hardly suitable for the transport of current. On the other hand, states such as $(2,1)$, when populated at finite gate voltages, move below $E_{F}$, thus losing their ability to carry current. 

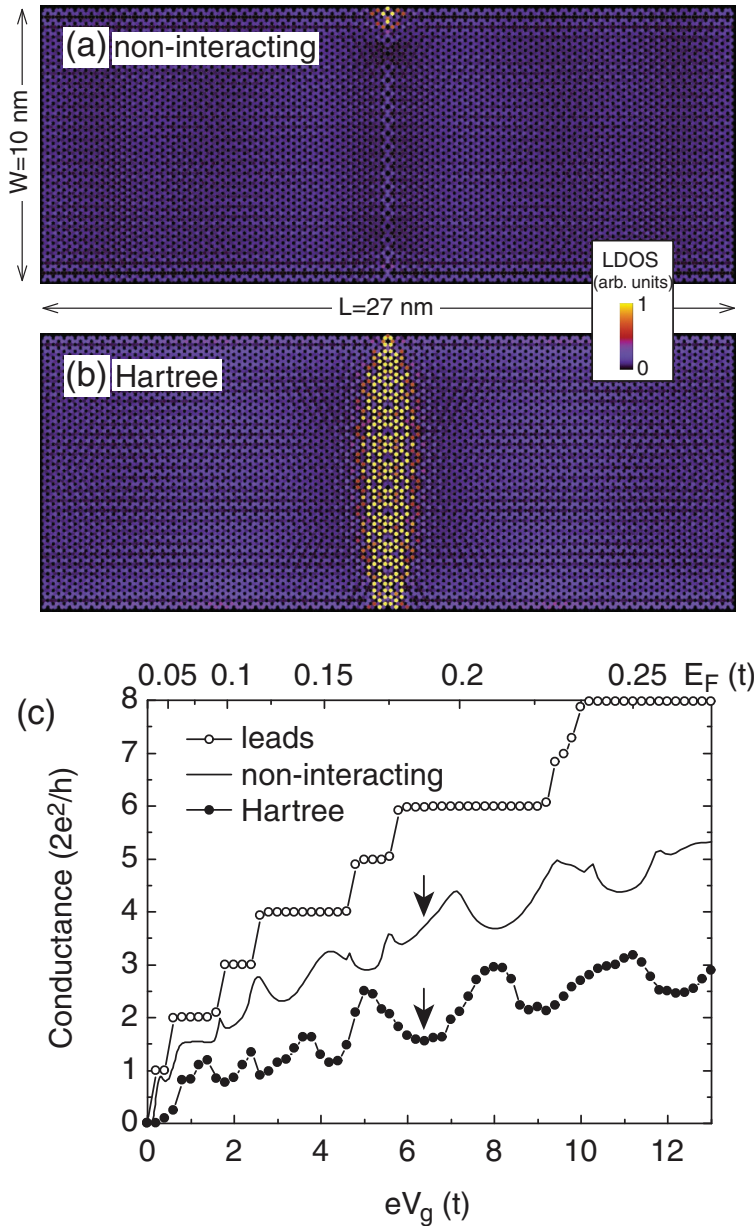

FIG. 4. (Color online) (c) The conductance of a graphene nanoribbon with the $(2,0)$ GB. The LDOS calculated in (a) the noninteracting model and (b) the Hartree model at $E_{F}=6.4 t$ [marked by arrows in panel (c)]. Ribbon's width $W=10 \mathrm{~nm}$; length $L=$ $27 \mathrm{~nm}$, which corresponds to 41 carbons in the transverse direction and 63 unit cells in the longitudinal direction.

To study the effect of the GB on electron transport we consider the $(2,0) \mathrm{GB}$ embedded in an armchair ribbon (see Fig. 4). The conductance of an ideal ribbon shows quantized steps corresponding to the opening of new transverse subbands [Fig. 4(c)]. The conductance of a ribbon with the GB calculated for noninteracting electrons shows an overall drop of $\sim 30 \%$ in comparison to the ideal case, and it exhibits an oscillating behavior resulting from electron interference within the GB. Accounting for electron interaction results in a further drop of the conductance $\left(\sim \frac{2}{3}\right.$ in comparison to the noninteracting case). The smaller conductance of the GB for interacting electrons in comparison to noninteracting ones is due to enhanced backscattering from the electrostatic potential at the GB caused by the electrons accumulated there [see the LDOS in Figs. 4(a) and 4(b)]. It is interesting to note that the Hartree approaches predict a somewhat larger transport gap in comparison to the noninteracting case.

Let us finally explore spin polarization in the GBs. It has been shown before that the $(2,0)$ GB in neutral graphene is spin polarized with the ferromagnetic ground state. ${ }^{16,17,21,22}$ Our calculations based on the Hamiltonian Eq. (3) show
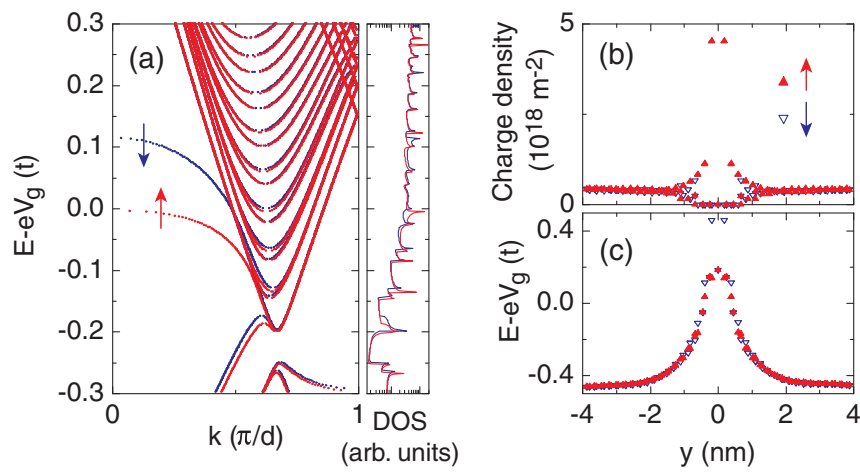

FIG. 5. (Color online) (a) The band structure, (b) the charge density, and (c) the self-consistent potential calculated in the Hubbard-Hartree approach for the $(2,0) \mathrm{GB}$ at $e V_{g}=5 t$.

that this state remains fully spin-polarized in electrostatically doped graphene at a finite $V_{g}$ with the electron population being completely dominated by species of the same spin; see Fig. 5 for the spin-resolved band structure, the electron density, and the potential of the $(2,0) \mathrm{GB}$ at $e V_{g}=5 t$, [compare with the spin-degenerate band structure of the same structure in Fig. 2(b)]. Due to the metallic character of the flat state $(2,0)$ which is partially filled at $E_{F}$, the electron density in this state can be easily redistributed. Hence, the spin-up and spin-down states can have different densities and thus experience different interactions due to the Hubbard term. As a result, the spin-down state is pushed up by the Hubbard interaction above $E_{F}$ and gets depopulated while the spin-up state remains populated and pinned to $E_{F}$. This is similar to the spin polarization of compressible strips in quantum wires and graphene nanoribbons in a high magnetic field. ${ }^{29,33}$

For the case of the $(2,1) \mathrm{GB}$, the corresponding state is fully occupied even for small applied $V_{g}$, and therefore the spin-up and spin-down electron densities are the same. As a result, the potential felt by different spin species is the same and the spin polarization for the $(2,1)$ state is completely suppressed.

\section{CONCLUSIONS}

We demonstrated that electronic, transport, and spin properties of GBs are strongly modified in electrostatically doped graphene at finite electron densities in comparison to a conventional noninteracting electron picture. Our calculations of the band structure and the conductivity were based on the self-consistent Green's function technique where electron interactions were included by the Hartree potential (for spinless electrons) and by the Hartree and Hubbard potentials (for the spin-resolved case). Our main findings can be summarized as follows.

(1) We demonstrated that the character of charge accumulation is different for different GBs. In particular, the charge density at the $(2,0)$ GB grows linearly with an increase of the gate voltage $V_{g}$. In contrast, the charge density at the $(2,1)$ GB stays practically constant, showing only a slow increase following the increase of the overall electron density in the ribbon as $V_{g}$ grows. We analyzed in detail the band structure 
and related the above difference in the charge accumulation to the different characters of the band dispersions and the DOS of the states residing at the $(2,0)$ and $(2,1) \mathrm{GBs}$.

(2) We calculated the numerically exact self-consistent potential using Hamiltonian Eq. (1) and showed that this potential can be satisfactory approximated by the analytical expression, Eq. (4), obtained within the semiclassical TF approximation.

(3) We studied the effect of the GB on electron transport by considering the $(2,0)$ GB embedded in an armchair ribbon. We demonstrated that accounting for electron interaction results in a drop of the conductance $(\sim 2 / 3$ as compared to the noninteracting case). We relate this to the enhanced backscattering from the electrostatic potential at the GB caused by the electrons accumulated there.

(4) In contrast to earlier speculations in the literature that GBs can be used as one-dimensional quantum wires to carry the current in bulk graphene, our findings suggest that these predictions might be overoptimistic. Even though the $(2,0)$ GB state retains its metallic character even at finite electron densities, its group velocity is practically zero, which makes this state hardly suitable for the transport of current. As far as the $(2,1)$ GB state is concerned, when populated at finite gate voltages it moves below $E_{F}$, thus losing its ability to carry current.

(5) For the spin-polarized case we found that in electrostatically doped graphene at a finite $V_{g}$ the state residing at the $(2,0)$ GB gets fully spin polarized with the electron population being completely dominated by species of the same spin. In contrast, $(2,1)$ GB states remain spin degenerate. This difference is traced to different characters of the band dispersions of these states.

\section{ACKNOWLEDGMENTS}

The authors gratefully acknowledge financial support from the Swedish Institute and thank George Kirczenow and Aires Ferreira for discussions.

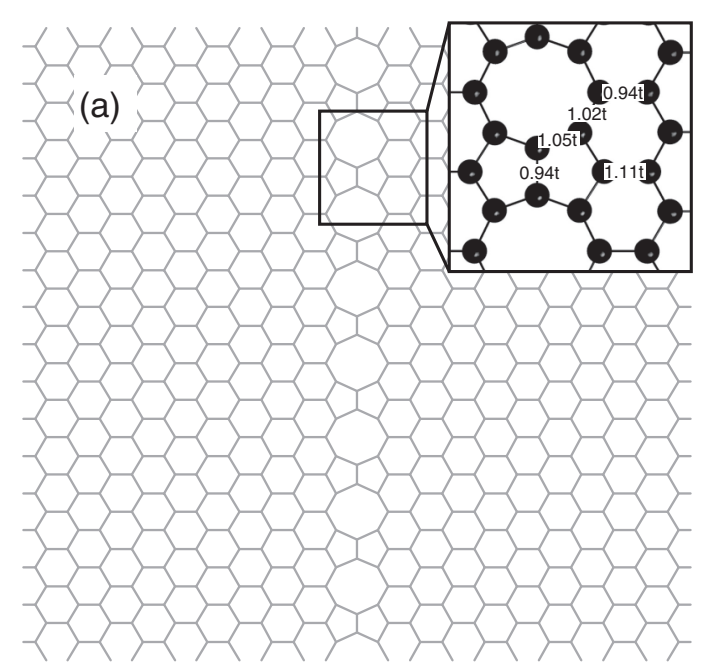

\section{APPENDIX: GEOMETRY RELAXATION AND CALCULATIONS OF THE HOPPING INTEGRALS}

We performed ab initio geometry relaxations based on the density functional theory for the grain boundary defects using the GAUSSIAN 09 software package. ${ }^{28}$ The relaxed geometries calculated in this way are expected to be accurate since the density functional theory has been well optimized for carrying out the accurate ground-state total energy calculations. ${ }^{34}$ The structures studied were graphene flakes of about a hundred of carbon atoms passivated at the edges with hydrogen with the grain boundaries being extended across the flake. The atoms of the grain boundary as well as the nearest carbon atoms were allowed to relax freely, while the other carbon atoms were allowed to move normal to the defect line; the whole geometry was kept planar. The relaxed structures obtained in this way are shown in the insets in Fig. 6.

The tight-binding model Hamiltonian, Eq. (1), includes modified hopping energies between the graphene carbon atoms calculated from the relevant matrix elements within the extended Hückel model. The extended Hückel theory is formulated in terms of small basis sets of Slater-type atomic orbitals $\left\{\left|\phi_{i}\right\rangle\right\}$, their overlaps $S_{i j}=\left\langle\phi_{i} \mid \phi_{j}\right\rangle$, and the Hamiltonian matrix $\mathcal{H}_{i j}=\left\langle\phi_{i}|\mathcal{H}| \phi_{j}\right\rangle$. The diagonal Hamiltonian elements $\mathcal{H}_{i i}=\mathcal{E}_{i}$ are chosen to be the experimentally determined atomic orbital ionization energies $\mathcal{E}_{i}$. In the present work the nondiagonal elements are approximated as in Ref. 35 by $\mathcal{H}_{i j}=\left(1.75+\Delta_{i j}^{2}-0.75 \Delta_{i j}^{4}\right) S_{i j}\left(\mathcal{E}_{i}+\mathcal{E}_{j}\right) / 2$, where $\Delta_{i j}=\left(\mathcal{E}_{i}-\mathcal{E}_{j}\right) /\left(\mathcal{E}_{i}+\mathcal{E}_{j}\right)$, a form chosen to reproduce experimental molecular electronic structure data. ${ }^{35}$ In the standard tight-binding Hamiltonian of pristine graphene, the energy scale is chosen such that the carbon $2 p_{z}$ orbital energy is zero, whereas in the extended Hückel theory ${ }^{35}$ the carbon $2 p_{z}$ orbital energy is the ionization energy $\mathcal{E}_{\mathrm{C}_{p_{z}}}=-11.4 \mathrm{eV}$. Accordingly, for consistency, in the extended Hückel Hamiltonian matrix we make the replacement $\mathcal{H}_{i i} \rightarrow \mathcal{H}_{i i}-\mathcal{E}_{\mathrm{C}_{p z}}$. Because the extended Hückel basis states on different atoms are not, in general, mutually orthogonal, the nondiagonal extended

FIG. 6. Relaxed atomic geometries with representative hopping energies in units of $t$ for $(2,0)$ and $(2,1)$ GBs [panels (a) and (b), respectively]. All geometries are in-plane. Grain boundaries separate two crystalline domains rotated by different tilt angles $\theta=0$ and $10.9^{\circ}$, panels (a) and (b), respectively.

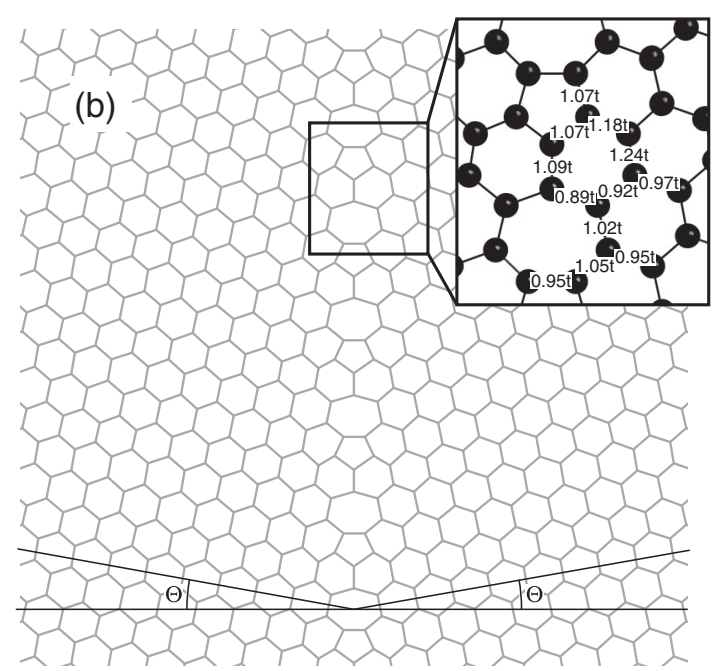


Hückel Hamiltonian matrix elements are then also adjusted according to

$$
\mathcal{H}_{i j} \rightarrow \mathcal{H}_{i j}-S_{i j} \mathcal{E}_{\mathrm{C}_{p z}} .
$$

Finally we extract the nearest-neighbor Hamiltonian matrix elements $t_{i j}$ from $\mathcal{H}_{i i}$ and incorporate them into the standard $\pi$-band Hamiltonian, Eq. (1), used for the self-consistent calculations. For consistency with the standard tight-binding model of pristine graphene we scale the values of $t_{i j}$ obtained from the extended Hückel model as described in Ref. 36. The inserts in Fig. 6 show the extracted hopping energies $t_{i j}$.

\section{*sihnatse@sfu.ca}

†igor.zozoulenko@liu.se

${ }^{1}$ K. S. Kim, Y. Zhao, H. Jang, S. Y. Lee, J. M. Kim, K. S. Kim, J. H. Ahn, P. Kim, J. Y. Choi, and B. H. Hong, Nature (London) 457, 706 (2009)

${ }^{2}$ X. Li, W. Cai, J. An, S. Kim, J. Nah, D. Yang, R. Piner, A. Velamakanni, I. Jung, E. Tutuc, S. K. Banerjee, L. Colombo, and R. S. Ruoff, Science 324, 1312 (2009).

${ }^{3}$ P. Y. Huang, C. S. Ruiz-Vargas, A. M. van der Zande, W. S. Whitney, M. P. Levendorf, J. W. Kevek, S. Garg, J. S. Alden, C. J. Hustedt, Y. Zhu, J. Park, P. L. McEuen, and D. A. Muller, Nature (London) 469, 389 (2010).

${ }^{4}$ K. Kim, Z. Lee, W. Regan, C. Kisielowski, M. F. Crommie, and A. Zettl, ACS Nano 5, 2142 (2011).

${ }^{5}$ For a review see, e.g., H. Terrones, R. Lv, M. Terrones, and M. S. Dresselhaus, Rep. Prog. Phys. 75, 062501 (2012).

${ }^{6}$ O. V. Yazyev and S. G. Louie, Nat. Mater. 9, 806 (2010).

${ }^{7}$ Q. Yu, L. A. Jauregui, W. Wu, R. Colby, J. Tian, Z. Su, H. Cao, Z. Liu, D. Pandey, D. Wei, T. F. Chung, P. Peng, N. P. Guisinger, E. A. Stach, J. Bao, S.-S. Pei, and Y. P. Chen, Nat. Mater. 10, 443 (2011).

${ }^{8}$ M. Ahmad, H. An, Y. S. Kim, J. H. Lee, J. Jung, S.-H. Chun, and Y. Seo, Nanotechnology 23, 285705 (2012).

${ }^{9}$ L. Tapasztó, P. Nemes-Incze, G. Dobrik, K. J. Yoo, Ch. Hwang, and L. P. Biró, Appl. Phys. Lett. 100, 053114 (2012).

${ }^{10}$ A. W. Tsen, L. Brown, M. P. Levendorf, F. Ghahari, P. Y. Huang, R. W. Havener, C. S. Ruiz-Vargas, D. A. Muller, P. Kim, and J. Park, Science 336, 1143 (2012).

${ }^{11}$ J. C. Koepke, J. D. Wood, D. Estrada, Z.-Y. Ong, K. T. He, E. Pop, and J. W. Lyding, ACS Nano 7, 75 (2013).

${ }^{12}$ A. Ferreira, X. Xu, C.-L. Tan, S.-K. Bae, N. M. R. Peres, B.-H. Hong, B. Ozyilmaz, and A. H. Castro Neto, Europhys. Lett. 94, 28003 (2011).

${ }^{13}$ J. N. B. Rodrigues, N. M. R. Peres, and J. M. B. Lopes dos Santos, Phys. Rev. B 86, 214206 (2012).

${ }^{14}$ D. V. Tuan, J. Kotakoski, T. Louvet, F. Ortmann, J. C. Meyer, and S. Roche, Nano Lett. 13, 1730 (2013).

${ }^{15}$ T. M. Radchenko, A. A. Shylau, I. V. Zozoulenko, and A. Ferreira, Phys. Rev. B 87, 195448 (2013).

${ }^{16}$ A. R. Botello-Méndez, E. Cruz-Silva, F. López-Urías, B. G. Sumpter, V. Meunier, M. Terrones, and H. Terrones, ACS Nano 3, 3606 (2009).
${ }^{17}$ S. S. Alexandre, A. D. Lúcio, A. H. Castro, and R. W. Nunes, Nano Lett. 12, 5097 (2012).

${ }^{18}$ D. A. Bahamon, A. L. C. Pereira, and P. A. Schulz, Phys. Rev. B 83, 155436 (2011).

${ }^{19}$ J. Song, H. Liu, H. Jiang, Q.-f. Sun, and X. C. Xie, Phys. Rev. B 86, 085437 (2012)

${ }^{20}$ L. Jiang, G. Yu, W. Gao, Z. Liu, and Y. Zheng, Phys. Rev. B 86, 165433 (2012).

${ }^{21}$ L. Kou, C. Tang, W. Guo, and C. Chen, ACS Nano 5, 1012 (2011).

${ }^{22}$ S. Okada, K. Nakada, K. Kuwabara, K. Daigoku, and T. Kawai, Phys. Rev. B 74, 121412(R) (2006).

${ }^{23}$ J. Lahiri, Y. Lin, P. Bozkurt, I. I. Oleynik, and M. Batzill, Nat. Nanotechnol. 5, 326 (2010).

${ }^{24}$ G. W. Semenoff, V. Semenoff, and F. Zhou, Phys. Rev. Lett. 101, 087204 (2008)

${ }^{25}$ J. Jung, Z. Qiao, Q. Niu, and A. H. MacDonald, Nano Lett. 11, 3453 (2011).

${ }^{26}$ Z. Qiao, J. Jung, Q. Niu, and A. H. MacDonald, Nano Lett. 12, 2936 (2012).

${ }^{27}$ A. A. Shylau, J. W. Klos, and I. V. Zozoulenko, Phys. Rev. B 80, 205402 (2009).

${ }^{28}$ GAUSSIAN 09, revision A.02 (Gaussian, Inc., Wallingford, CT, 2009); The HSEh1PBE hybrid density functional and 6-311G(d) basis set were used in the geometry relaxations carried out in the present study.

${ }^{29}$ S. Ihnatsenka and I. V. Zozoulenko, Phys. Rev. B 86, 155407 (2012).

${ }^{30}$ S. Ihnatsenka and G. Kirczenow, Phys. Rev. B 86, 075448 (2012).

${ }^{31}$ T. O. Wehling, E. Şaşioğlu, C. Friedrich, A. I. Lichtenstein, M. I. Katsnelson and S. Blügel, Phys. Rev. Lett. 106, 236805 (2011).

${ }^{32}$ O. V. Yazyev, Phys. Rev. Lett. 101, 037203 (2008).

${ }^{33}$ S. Ihnatsenka and I. V. Zozoulenko, Phys. Rev. B 78, 035340 (2008).

${ }^{34}$ For a recent review see G. Kirczenow, in The Oxford Handbook of Nanoscience and Technology, Volume I: Basic Aspects, edited by A. V. Narlikar and Y. Y. Fu (Oxford University Press, London, 2010), Chap. 4.

${ }^{35}$ The version of extended Hückel theory that we use here is that of $\mathrm{J}$. H. Ammeter, H.-B. Bürgi, J. C. Thibeault, and R. Hoffman, J. Am. Chem. Soc. 100, 3686 (1978), as implemented in the YAEHMOP numerical package by G. A. Landrum and W. V. Glassey (SourceForge, Fremont, California, 2001).

${ }^{36}$ S. Ihnatsenka and G. Kirczenow, Phys. Rev. B 83, 245442 (2011). 